\section{Hirnmetastasen bei Brustkrebs: Vorteile für TKI}

Der molekulare Subtyp von Mammakarzinomen beeinflusst auch bei ZNSMetastasen über die Prognose. Das ergab eine retrospektive Studie, in der verschiedene Therapieoptionen analysiert wurden.

n der Auswertung wurden 547 Patientinnen mit bekanntem Hormonrezeptorund HER2-Status berücksichtigt. Sie hatten Hirnmetastasen entwickelt und eine Ganzhirnhirnbestrahlung, stereotaktische Radiochirurgie (SRS) und/oder

Operation erhalten; die meisten danach

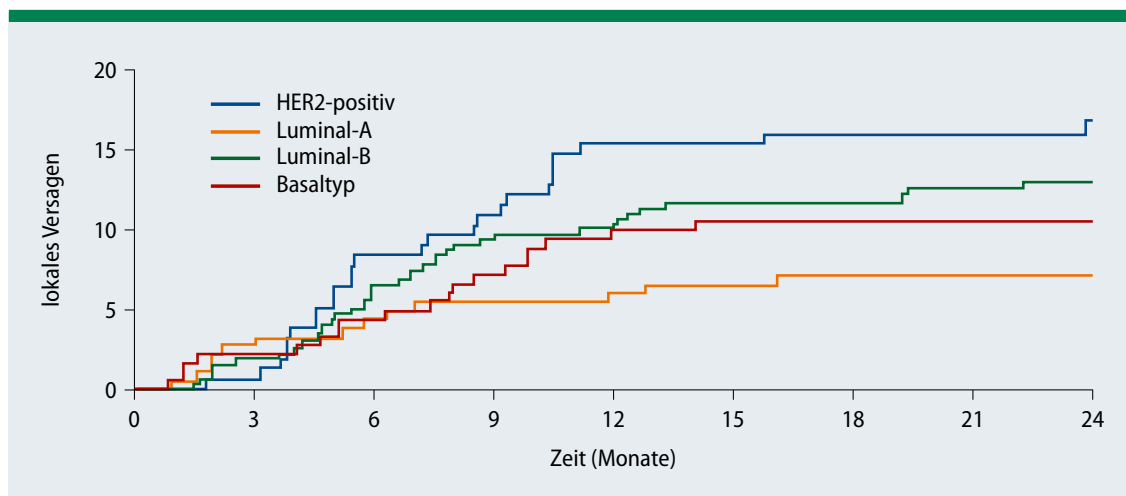

Abb. 1: Die kumulative Inzidenz eines lokalen Versagens erwies sich als abhängig vom molekularen Subtyp.

\section{Nodal positives Mammakarzinom: AC-freie Regimes versus Standardchemotherapie}

\section{Doxorubicin plus Cyclophosphamid (AC) gefolgt von Paclitaxel ist in der adjuvanten Chemotherapie des nodal positiven Mammakarzinoms ein Standard. Welchen Nutzen bieten AC-freie Regimes und/oder Docetaxel?}

$E_{s}^{\text {in }}$ in Standard bei Frauen mit nodal positivem Brustkrebs sind adjuvant 4 Zyklen AC, gefolgt von 4 Zyklen Paclitaxel. Das Optimum ist hier aber möglicherweise noch nicht erreicht, und es war bisher nicht bekannt, ob Docetaxel in diesem Kontext vielleicht wirksamer ist als Paclitaxel. Beide Fragestellungen wurden in einer Phase-III-Studie aufgegriffen. Randomisiert erhielten 1.049 Frauen mit nodal positivem Brustkrebs postoperativ entweder 4 Zyklen AC, gefolgt von 4 Zyklen Paclitaxel (ACpT) oder AC gefolgt von 4 Zyklen Docetaxel auch eine Hormontherapie. $80 \%$ der Patientinnen mit HER2-positiven Tumoren bekamen HER2-Antikörper (meist Trastuzumab) und $38 \%$ HER2/EGFR-Tyrosinkinasehemmer (TKI, z. B. Lapatinib).

Beim primären Endpunkt Gesamtüberleben (OS) ergaben sich signifikante Unterschiede - mit median 8,4 Monaten beim Basal-, 12,3 Monaten beim Luminal-A-, 15,4 Monaten beim HER2-positiven und 18,8 Monaten beim Luminal-BTyp ( $\mathrm{p}<0,001)$. Bei Frauen mit HER2-positivem Tumor verlängerten HER2-Antikörper (17,9 vs. 15,1 Monate; $\mathrm{p}=0,04$ ) und mehr noch TKI (21,1 vs. 15,4 Monate, $\mathrm{p}=0,03)$ das Überleben. Auch die kumulative 12-Monats-Inzidenz für lokalen Progress variierte je nach Tumorsubtyp (Abb. 1). Bei stereotaktischer Bestrahlung und HER2/EGFR-TKI ging sie von 15,1 auf 5,7\% (p < 0,001) zurück.

Fazit: Bei Brustkrebspatientinnen mit Hirnmetastasen ist der molekulare Subtyp ein wichtiger Prognosefaktor und prädiktiv für das Ansprechen auf eine Strahlentherapie. Anti-EGFR-TKI verbessern bei HER2-positiven Tumoren OS und lokale Kontrolle und stellen zusammen mit einer SRS eine gute Option dar.

Barbara Kreutzkamp

Miller JA et al. Overall survival and the response to radiotherapy among molecular subtypes of breast cancer brain metastases treated with targeted therapies. Cancer. 2017;123(12):2283-93.

(HR 0,$72 ; p=0,0008)$ sowie auch ein längeres Gesamtüberleben (HR 0,75; p = 0,035). Unter AC traten Neutropenie, Übelkeit und Erbrechen häufiger auf, unter Docetaxel Ödeme. Insgesamt erwies sich die Toxiziät von Docetaxel als akzeptabel und handhabbar.

Fazit: Bei Frauen mit nodal positivem Brustkrebs kann ein AC-Schema nicht ohne weiteres durch ein AC-freies ausgetauscht werden. Bei der Taxan-Anschlussbehandlung ist das DFS unter Docetaxel höher als unter Paclitaxel. Docetaxel könnte damit eine brauchbare Alternative bieten. Barbara Kreutzkamp

Beim primären Endpunkt krankheitsfreies Überleben (DFS) ließ sich keine Nichtunterlegenheit von AC-freien gegenüber AC-haltigen Behandlungen zei-

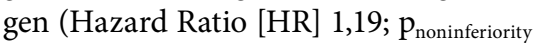
$=0,30)$. Patientinnen hatten unter Docetaxel-haltiger Therapie ein längeres DFS als jene mit Paclitaxel-haltigen Therapien
Watanabe T et al. Comparison of an AC-taxane versus $A C$-free regimen and paclitaxel versus docetaxel in patients with lymph node-positive breast cancer: Final results of the National Surgical Adjuvant Study of Breast Cancer 02 trial, a randomized comparative phase 3 study. Cancer. 2017;123(5):759-68. 